\title{
An automated non-destructive testing system for seam bondings in automotive serial production
}

\author{
Maximilian Klausing ${ }^{1 *}$ and Henning Heuer ${ }^{2}$
}

*Correspondence:
maximilian.klausing@
tu-dresden.de; maximilian.
klausing@volkswagen.de
${ }^{1}$ Division Technology
Management, Volkswagen
AG, 38440 Wolfsburg,
Germany
Full list of author information
is available at the end of the
article

${ }^{*}$ Correspondence: maximilian.klausing@ klausing@volkswagen.de 1 Division Technology Management, Volkswagen AG, 38440 Wolfsburg, Full list of author information article

\begin{abstract}
The joining process of seam bond is used in automobile construction because of the non-visible joints on mounted parts such as doors, hoods or luggage compartment doors. With these components, a structure-providing inner panel is joined with the outer panel, which is visible to the customer. Besides providing stability, particularly in the event of a crash, the joining level is also filled with adhesive to increase the stability and to prevent the ingress of moisture that causes corrosion. These mechanical connections must be tested to ensure that the requirements for proper bonding have been met. As the destructive test used today requires metallographic laboratory with special trained personnel, significant scrap cost and a time consuming process control loop, considerable efforts are being made to develop a capable non-destructive and as far as possible in-line test method. State of the art non-destructive inspection systems for seam bonds are insufficient regarding full part accessibility and reliability in terms of detectability and validation. This paper is focussed on new developed free configurable three-transducer ultrasonic phased array technology including algorithms for data validation and evaluation for an automated process control loop in the automotive car body shop. The paper end with an statistical classification based on an 5-month measurement campaign under industrial in-line conditions. In a first step, a list of possible defects was collected and the detectability in different seam areas was calculated by sound field simulation of the amplitude signals. Second, based on the results from simulation, a measurement setup as excitation sequence was defined. Based on the theoretical findings a inspection system was designed and realized. Within a measurement campaign the inspection system was evaluated in terms of detectability of minimal defect size under non-laboratory conditions. This includes a permanent proof of coupling conditions, monitoring of misalignments, e.g. tilting and surface defects of the car body. In the last step, the testing system is build up and tested within the assembly line. These measurement results show that the identification of common process defects is possible.
\end{abstract}

Keywords: Non-destructive testing of seam bondings, Phased array ultrasonic

\section{Background}

Seam bonding is used in automotive engineering in particular because of the high degree of automation and, in the case of the table-top seam bonding process, because of the very fast cycle times that are achieved. This hybrid joining process plastically bends 
the sheet metal edge of the outer part around the inner part producing a form and forcelocking joint $[1,2]$. The gap between the two sheet metal parts is filled with adhesive. This bonding firstly prevents the ingress of moisture at the joint level, and secondly the adhesive provides the bond with greater bending and torsional rigidity [3]. The fact that this joining method, in contrast to welding, does not leave any visible evidence of the connection, allows it to be used in areas where the joint is visible. In the automotive industry, this benefit makes the process particularly suitable for use for the closures (e.g. doors, hood top, luggage compartment door) where seam bonding is used to combine the visible outer skin parts with the inner structure and crash resistant parts.

Various influences can cause a defect in the seam bonding. A displacement of the inner to the outer sheet inevitably leads to a lack of adhesive in the seam areas [4, 5], a spring back of the outer sheet directly after the hemming process provokes meander structures [5-7]. Other defects, which influence the geometrical shape, are described in [8]. The new standard DIN EN 2304-1 [9] to ensure the quality requirements of bonding processes stipulates that the process must be monitored. The aim is to guarantee consistent process quality. The specified testing interval is short because the joints are crash-relevant. Of particular interest in the uncured adhesive condition is the degree of filling in the areas a, b, c and d (see Fig. 1, left). Currently this involves a destructive test by physically opening the seam [10]. This type of destructive test takes about $8 \mathrm{~h}$, and is therefore not performed each day. This causes a long process control loop. A non-destructive test has the potential to save money by avoiding scrap and personnel costs and ensuring the quality of all the seam bond produced. This encourages the development of a capable non-destructive inline testing system.

Non-destructive inspection systems based on X-ray, Thermography and ultrasonic are commercial available for this task. A Volkswagen intern evaluation of this systems pointed out that no existing non-destructive testing system can solve the requirements for automated inline inspection. In the case of X-ray, there is possibility to detect small air gaps. Furthermore, this system has high demands on radioprotection. The Thermography has limited resolution and the result is difficult to interpret because of the many parasitic effects. Available systems based on ultrasonic phased array in impuls echo [11] or transmission mode [12] (wheel probes, probes with an elastic wedge) as well as single element sensors [13] are not useable for the inspection task of all three seam areas. Seam areas b and $c$ are especially difficult to test due to their size and form. In addition, the process control loop for hand-guided inspection is not much shorter than the destructive test. Therefore a solution based on a multi probe phased array ultrasound system with a free configurability of different transmission and reflection measurements

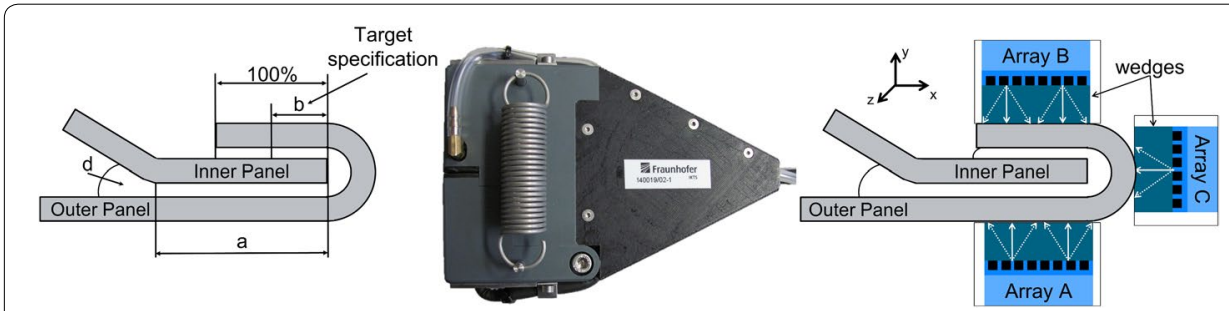

Fig. 1 Schematic representation of a seam bonding (left), prototype of the multi phased array system (middle), schematic of the ultrasonic test system (right) 
was proposed for an automated evaluation of the seam areas a, b and c (see Fig. 1) as an inline system in the automobile car body production. A VW standard requires that these areas achieve different degrees of adhesive filling. Seam areas a and $b$ should be filled with a 100 and $30 \%$ adhesive respectively [14]. These filling degrees are both measured from the seam loop c. In the seam loop c the entire surface of the sheet metal must be filled with adhesive.

For the ultrasound investigation of bonds, well-known manufacturers offer a wide variety of ultrasonic transducers. However, all systems are currently hand-guided designed for the testing of even bondings or just for seam area a. Inline systems for testing seam bondings are not available. Despite the fact that the testing of all seam bond areas directly in the production line offers the opportunity to shorten the process control loop effectively.

For the testing of even adhesive bondings ultrasonic systems, such as single probes and phased array systems, are commonly discussed in the literature [15-17]. These handheld systems are not sufficient for industrial inline applications. The transmission from manual guided probes to fully automated inspection requires an integrated coupling procedure, test condition proof and a accessibility of curved parts regions. A tolerance against obstacles e.g. geometry irregularity is like ridge or wrinkles at curvatures is necessary to prevent damages of sensors or the part. Due to these requirements, processes and devices that are proofed for manual inspection cannot be transferred to automated inspection. Especially the testing for the seam area $\mathrm{c}$ is not provided by any standard ultrasonic systems. In a first VW intern valuation with standard phased arrays or single ultrasonic probes available on the market, a sufficient repeating accuracy and a satisfactory defect detectability could not be established for the seam areas a and $b$. The reasons for this, in case of the desired automated test process, are:

- Tilting of the seam bonding in the sensor.

- Different radii of the folding loop C within one component.

- Uneven surface of the outer sheet.

- Fluctuating adhesive layer thicknesses.

- Consistent quality of the ultrasound coupling.

- No possibility of amending the robot path because of component deviations.

Therefore, new evaluating-algorithms as well as a new signal processing are necessary in order to react to these challenges of a inline inspection system. In this document attention is paid to two of these challenges by means of simulations and real measurements. On the one hand, the tilting of the seam in the sensor, on the other hand the evaluation method for the defect detectability in the seam areas a, b and $\mathrm{c}$ are presented.

\section{Methods}

\section{Sound field simulation}

Model design

The sound propagation of the simulation model is calculated using the finite element method with the acoustics module of the COMSOL Multiphysics 5.2 program [18]. The simulation model is a two-dimensional model. The reduction in dimensions is necessary 
to decrease the degrees of freedom otherwise the computation times would increase to an unreasonable high. The derived model with the material assignments is shown in Fig. 2. For the area of the adhesive material the damping properties of the material on the wave are also considered. This characteristic is implemented by applying viscoelastic damping with the parameters of Bitumen [19]. In solid bodies, Eq. 1 is solved for each cross-linked element using the displacement field $\vec{u}$. This vector field has components in $\mathrm{x}$ and $\mathrm{y}$ direction for the special case of a two-dimensional simulation. To solve these model ranges, the density $\rho$ and the direction-dependent material stress $\sigma$ derived from the elastic modules and the Poisson number are required.

$$
\rho \cdot \frac{\partial^{2} \vec{u}}{\partial t^{2}}+d \frac{\delta \vec{u}}{\delta t}-\vec{\nabla} \sigma=0
$$

For each cross-linked element in the liquid area of the coupling, a differential wave equation for the scalar pressure $\mathrm{p}$ is solved as a function of the sound velocity $\mathrm{c}$ of the material at any time $t$.

$$
\Delta p=\operatorname{div} \operatorname{grad}(p)=\frac{1}{c^{2}} \cdot \frac{\partial^{2} p}{\partial t^{2}}
$$

At the boundary between the acoustic pressure and the linear-elastic regions, the force component of the pressure $\mathrm{p}$ from the fluid area perpendicular $\vec{n}$ to the surface is converted into an acceleration component perpendicular $\vec{n}$ to the surface of the displacement $\vec{u}$ in the solid body. This process is independent of direction.

$$
\vec{n} \cdot \frac{\partial^{2} \vec{u}}{\partial t^{2}}=\vec{n} \cdot\left(\frac{1}{\rho} \vec{\nabla} p\right)
$$

In the simulation, the piezoelectric effect is not considered for the excitation and the detection of the ultrasonic waves because of missing material parameters. Both are realized through a pressure condition respectively pressure detection on the boundaries of the three wedges, where the piezo transducers are located. Considering the arrays A or $\mathrm{B}$, the following results are obtained as a function of the location $\mathrm{x}$ on the wedge. All

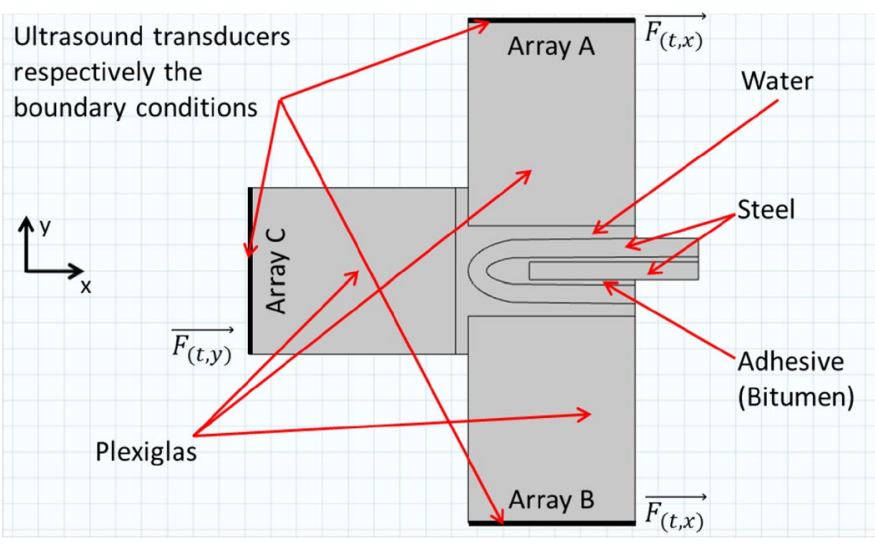

Fig. 2 Schematic of the 2D simulation model 
three transducers are composed of 16 piezo transducers with a width b of $350 \mu \mathrm{m}$ and a distance a between adjacent elements of $50 \mu \mathrm{m}$

$$
h(x)={ }_{0}^{1} \text { if } \begin{gathered}
{[a i+b \cdot(i-1)] \leq x \leq(a+b) i} \\
\text { otherwise }
\end{gathered}
$$

For the stimulation of the ultrasonic wave a sinusoidal oscillation is scaled with a Gaussian. The frequency $f$ of the sinusoidal oscillation is no longer a harmonic oscillation due to the superposition with the Gaussian bell curve. Rather, a frequency spectrum with a mean frequency $f$ is generated by this superposition. This corresponds to a stimulation of real oscillators.

The detection of the simulated ultrasonic amplitude signals is calculated from the path integral of the deflections $\vec{u}$ in the direction of the surface normals in the oscillation range at each simulated time $t$. Therefore, the temporal signals represent the surface of the respective transducer element, which is deflected against the rest position. For the special case that the elements are arranged in the array in the $\mathrm{x}$ direction (array A and B, see Fig. 2), the amplitude signal $s_{i, j}(t)$ for element $i$ with the width $b$ of the array $j$ at the time $t$ can be calculated as:

$$
s_{i, j}(t)=\int_{x_{i, j}-b / 2}^{x_{i, j}+b / 2} \overrightarrow{u_{(t)}} \times \overrightarrow{\delta x} \text { with } \overrightarrow{u_{(t)}}=\begin{aligned}
& u_{x(t)} \\
& u_{y(t)}
\end{aligned} ; \quad i=1,2, \ldots 16 ; \quad j=1,2
$$

The model is solved in the time range, whereby both, a spatial solution using the crosslinked elements and a temporal solution using the maximum time steps must be defined. The model is cross-linked using triangular elements, where the maximum element size $\mathrm{e}_{\max }$ corresponds to one tenth of the longitudinal wavelength of the respective material. The area of the ultrasonic transducers is again more finely cross-linked by a factor of 0.5 in order to detect the slowly propagating surface waves. To depict the temporal propagation process of the waves, the maximum permitted time steps $\Delta t_{\max }$ are predetermined for the solution of the differential equations, whereby the actual temporal wave propagation process is discretised. The Eq. 6 indicates that for a good temporal representation a maximum of $20 \%$ of a cross-link element of size e is traversed in one time step.

$$
\Delta t_{\max }=0.2 \cdot \frac{e_{\max }}{c}
$$

\section{Real measurements}

\section{Scope of the studies}

To determine the suitability of the ultrasound examination, various methods are used to validate the results of the nondestructive testing system. Thus, the results of the phased array system are matched with X-ray computer tomography as well as destructive tests. Because of the manufacturing process of seam bonds, the reliable preparation of artificial damages with adhesive is not possible. The adhesive is therefore replaced for the assessment and qualification of the error recognition. A Viton foil is used in its place, which has similar acoustic properties like the adhesive.

This paper shows and discusses the results of the study to estimate the minimal error recognition for the seam areas a and b. In addition, these measurements are used to verify the benefits of the new evaluation criterion for the detection of defects to the 
conventionally used method. The ultrasound result of an inspection of seam area $\mathrm{c}$ is compared with a destructive test. In addition, results of real luggage compartment doors, which are recorded directly in the production line, are presented and discussed.

\section{Test equipment}

In a first step, the freely configurable multi probe phased array was experimentally proofed in a feasibility study in close cooperation with the Fraunhofer Institute for Ceramic Technologies and Systems Dresden (IKTS). In the second step, an industrial prototype based on the IKTS PCUS ultrasonic platform was developed. For experimental validation, the inspection is done at a 1-component adhesive before the part is cured in the drying oven and a first coat of paint has been applied. Therefore, the test procedure evaluates the distribution of the un-hardened adhesive before curing takes place. As a result, all fabricated parts can be tested and the process loop is maximally shortened. In a last step, the testing system is adjusted for the industrial environment and integrated into an industrial production line.

The folding process in the automobile industry has to be simplified in order to obtain a general model for master samples. These probes are used to validate the ultrasonic system with defined errors. To do this, specimens were defined that have a sufficiently long $(150 \mathrm{~mm})$ and straight seam line. The flange length produced is $8 \mathrm{~mm}$ (seam bonding area a and c) and therefore within the limits of $4 \mathrm{~mm}$ to $12 \mathrm{~mm}$ for typical seam bond flange length [20]. According to the technical drawing, the geometrical dimensions of the seam bond are two adhesive layers of $200 \mu \mathrm{m}$ and two steel sheets of $700 \mu \mathrm{m}$ thickness. Glass spheres of a defined size are mixed together with the adhesive to ensure the correct adhesive layer thickness [21]. Because of the low percentage by volume of $2 \%$ the effect of the glass spheres on the ultrasonic wave is negligible. Both the inner and outer sheet metal panels have two holes cut by laser to use as reference points for fixing the position of the metal sheets in the folding tool. For estimating the minimum defect sizes and defect classes [7] that can be detected by the system, the seam bond has been produced with a Viton foil in place of the adhesive in seam area a and b. This film, which has similar acoustic properties as the adhesive, has laser-perforated holes of various different diameters ranging from 1 to $6 \mathrm{~mm}$. The overlapping of these defects is excluded by the minimum of $9 \mathrm{~mm}$ distance between the individual holes. This distance is greater than the length $(8 \mathrm{~mm})$ of the ultrasonic transducers used. The use of the Viton foil instead of the adhesive is necessary since the real manufacturing process cannot produce defects with defined dimensions.

The probe with the arrangement of the three ultrasound arrays on the seam bond is shown in Fig. 1 on the right. The gap between the ends of the two leading wedges of array $A$ and $B$ is determined to be $3.5 \mathrm{~mm}$, whereby array $C$ is predominantly obscured by arrays $A$ and $B$. An arc phased array $C$ is not possible because of the different radii of the seam loop within one mounting part. Water is continuously fed between the seam and the leading wedges as a couplant. Any fluctuations in the thickness of the seam bond pack size in the y direction (see Fig. 1) are to be compensated by the water layer or the articulated joint of the ultrasonic test system, which has a plier-like design.

Three ultrasound arrays provided by IKTS each consisting of 16 elements, a centre frequency of $5 \mathrm{MHz}$, an element pitch of $400 \mu \mathrm{m}$, an element width of $350 \mu \mathrm{m}$ and an 
element length of $8 \mathrm{~mm}$ are arranged for the ultrasonic testing of the three areas of the seam bond as shown in Fig. 1 on the right. This arrangement enables arrays A and B to achieve special resolution of $400 \mu \mathrm{m}$ when evaluating the adhesive in the $\mathrm{x}$-direction using the pulse echo method. The arrays A and B can inspect an area of $6.5 \mathrm{~mm}$ each, whereby the resolution for one test position consists of 14 individual measurements. This is done through a line scan with a 3 element aperture that is shifted by one element after every shot. This test area is sufficient for the complete testing of seam bond area $b$, whereas seam bond area a is usually larger. This means that this array cannot completely test seam bond area a. Array $\mathrm{C}$ can focus the ultrasound in the $\mathrm{y}$-direction to the strong curved edge. The ultrasonic transducers are controlled by a PCUS from Fraunhofer IKTS. This allows 64 individual channels to be controlled by the quadruple multiplexing of the 16 physical channels. Based on the PCUS Pro II with 48 (up to 128) native channels a system can be set up without multiplexing.

As ultrasound control and evaluation software the PCUS proLab suite from Fraunhofer IKTS is used. This software enables several phased array methods to be combined in a single cycle. This allows the seam bond areas a, b and c to be tested successively using a trigger system actuated by a linear encoder. As a major advantage of this configuration all three areas of the seam bond can be tested in one pass of the multi probe sensor.

Due to the complex 3 dimensional shape of typical car body parts, the inspection process requires the use of a robot for object manipulation. This passes the part under test through the sensor. The centre point of the sensor is integrated as an external tool on the robot, so that the part is moved through the sensor at a constant speed, which is independent of the geometrical shape of the component. The speed of travel is because of a sufficient stability of the water layer $30 \mathrm{~mm} / \mathrm{s}$.

\section{Results}

\section{Sound field simulation}

\section{Simulated evaluation of seam areas $a$ and $b$}

The arrays A and B are operated in pulse echo mode. An ultrasonic A scan resulting from the excitation of three adjacent elements and an averaged detection is shown in Fig. 3 for a section of seam bond completely filled with adhesive and as a second example filled with air. The largest captured echo is normalised to $70 \%$ of the total scale, whereby the y axis has no unit. Three echoes or echo sequences can be identified in the A scan. The echo between 7 and $8 \mu$ s comes from the interface between the Plexiglas wedge to water. The following echo in the range between 8 and $9 \mu$ s is caused by the transition from water to steel. The intermediate time between these two echoes corresponds with the speed of sound in water to the double water layer path, whereby in the real measurement this distance can fluctuate due to the undefined thickness of the water layer. From approximately $9.5 \mu \mathrm{s}$ an echo repetition is detected. The time intervals of which correspond to the double material thickness of the steel sheet. The amplitudes of these echo repetitions decrease with each oscillation period. From the two amplitude images it can be observed that the echo repetitions in the rear area (from $9.5 \mu \mathrm{s}$ ) are more strongly dampened by the adhesive present. 


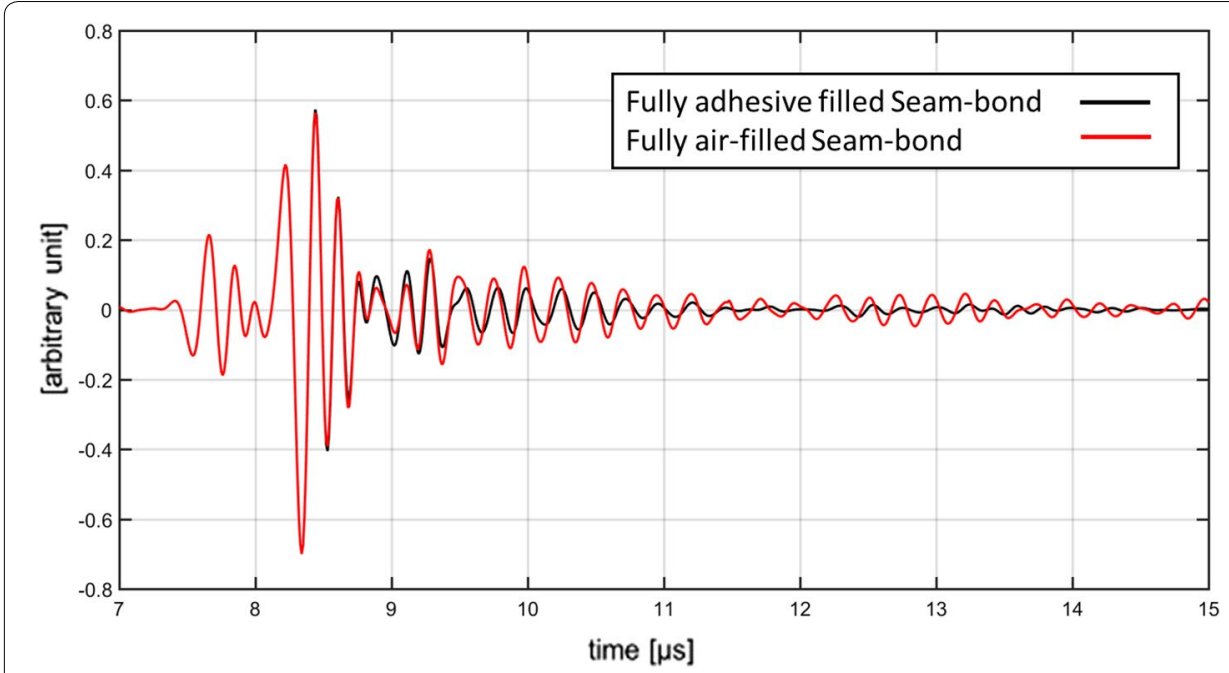

Fig. 3 Simulated amplitude scan of a flat, unfocused wave on a fully adhesive-filled seam-bond (black line) and on a seam bond completely filled with air (red line) (transmitter array A elements 7-9, receiver array A elements 7-9)

The signal wave-form shown in Fig. 3 is evaluated in gate $\mathrm{G}$ from 11 to $15 \mu \mathrm{s}$. The temporal range of $\mathrm{G}$ is a compromise between the stability of the signal and the discrepancy between the results of the integral energy method for a seam bond completely filled with air and with adhesive. In the rear temporal area of the amplitude scan, where the discrepancy in the simulations is highest, the usable signal and interferer becomes in real measurements similar. Two evaluation methods for generating the $\mathrm{C}$ image are presented The first is the conventional amplitude evaluation method. For that method the use of a time corrected gain curve is necessary. The TCG is used to compensate the travel time depending on signal attenuation. Without TCG the early, close to the surface signals will dominate the evaluation process, so that information form deeper areas have a minor influence for C-image evaluation. The other new-programmed evaluation method is the summation of all the measured values detected in the gate, which represents the oscillation energy within the gate G. A TCG compensation is not required when evaluating the oscillation energy with this new method. The oscillation energy $A_{i}$ from the oscillating element $i$ is processed by using Eq. 1 . All the magnitudes of the sampled amplitude signals s from the piezo transducer i are added within the gate $\mathrm{G}$. The summation of the sampled signals in Eq. 7 is shown by a number of Dirac-impulse $\delta$ with a similar sampling rate $\mathrm{T}$ like the time steps in the simulation. The resulting data is displayed as a percentage of a predefined maximum default oscillation energy to create the corresponding colour pixel representation in the $\mathrm{C}$ image. The predefined maximum is selected by the user based on the highest expected value.

$$
A_{i}=\left|s_{i}(t)\right| \cdot \sum_{n=G_{1}}^{G_{2}} \delta(t-n T) \text { with } T=\frac{1}{f_{\text {sampling }}} ; \quad G_{A, B}=\begin{aligned}
& 11 \mu \mathrm{s} \cdot f_{\text {sampling }} \\
& 15 \mu \mathrm{s} \cdot f_{\text {sampling }}
\end{aligned}
$$

Figure 4 show the defects as well as the results, when evaluating the signal bx the amplitude and the oscillation energy method. For better comparability, the maximum 


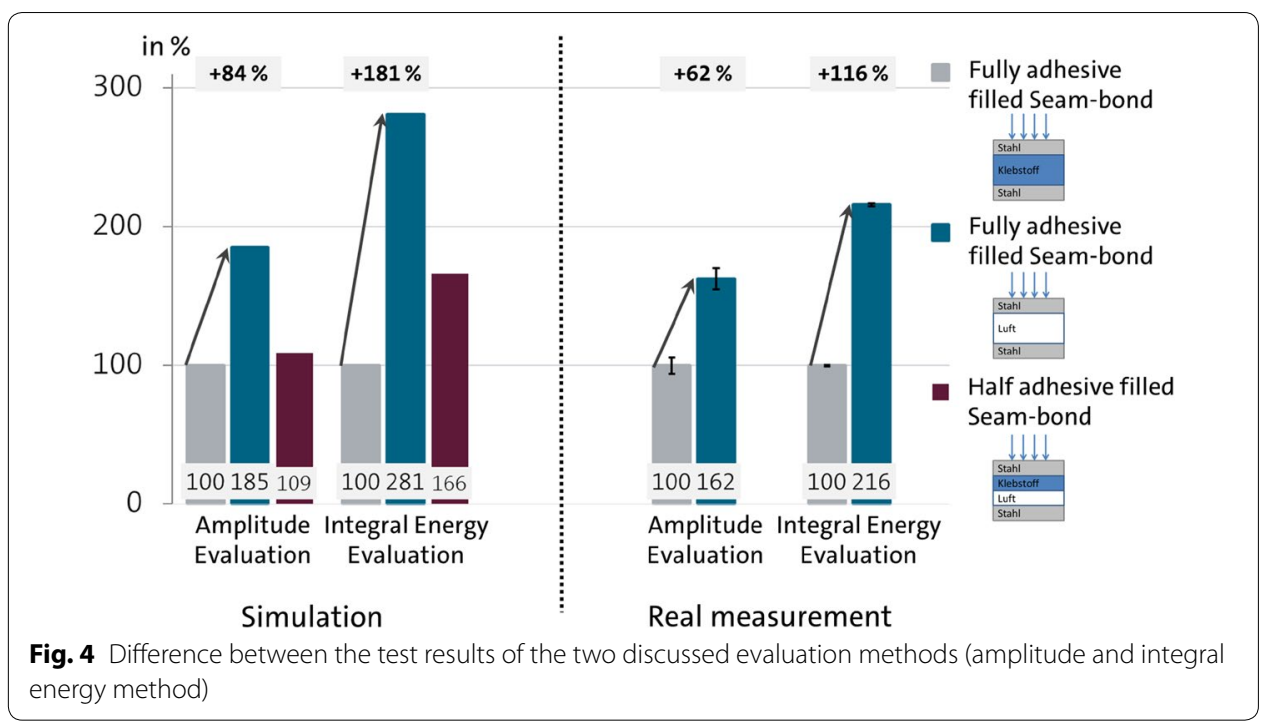

amplitude or the evaluated ultrasonic oscillation energy of a seam bond filled with adhesive has been set to $100 \%$. In contrast, the amplitude or the integral energy is expressed as a percentage in the presence of two simulated defect locations. This means that the deviation between a defective and an OK bonding in the case of the amplitude evaluation is $85 \%$ (see Fig. 4, left). In contrast, there is a difference of $181 \%$ when using integral energy evaluation. When evaluating the simulation of a half filled seam bond with adhesive, in which the adhesive is on the outer sheet, the deviation lies at $66 \%$. This type of defect cannot be identified as a defect when using amplitude evaluation due to the associated deviations of $9 \%$. In real measurements, this discrepancy can be the result of measurement fluctuations. This simulation result verified the improvement of the integral energy evaluation method [11, 22].

\section{Simulated evaluation of seam loopc}

The evaluation of the $\mathrm{C}$ area is more complicated than the evaluation of the planar areas $a$ and $b$. Due to design criteria, the seam loop runs to a point, whereby an elliptical seam loop can no longer be assumed in the proper sense. In addition, both the radius and the position of the seam loop in the in the test arrangement (see Fig. 1, right) can be assumed to not be uniform in the series production. However, simulations show that both parameter have a influence of the resulting A-scan.

The method of focusing array $\mathrm{C}$ on the seam loop does not show any significant deviation in the amplitude signal between a loop with a defect and a loop completely filled with adhesive. The sound field simulations can be used to discover the reasons for this effect. In Fig. 5 the time variation in the displacement value is shown for three time points before and after the impact of the focused ultrasonic wave on seam loop. The focus point of the ultrasonic wave is on the cut edge of the inner sheet metal. This simulation reveals that hardly any sound energy is introduced into the sheet-to-adhesive interface because of the curvature of the seam loop. Rather, the curvature reflects the incoming ultrasonic wave into the outer sheet, whereby two wave packets are formed in the outer sheet. These wave packets propagate within the sheet metal, where they 


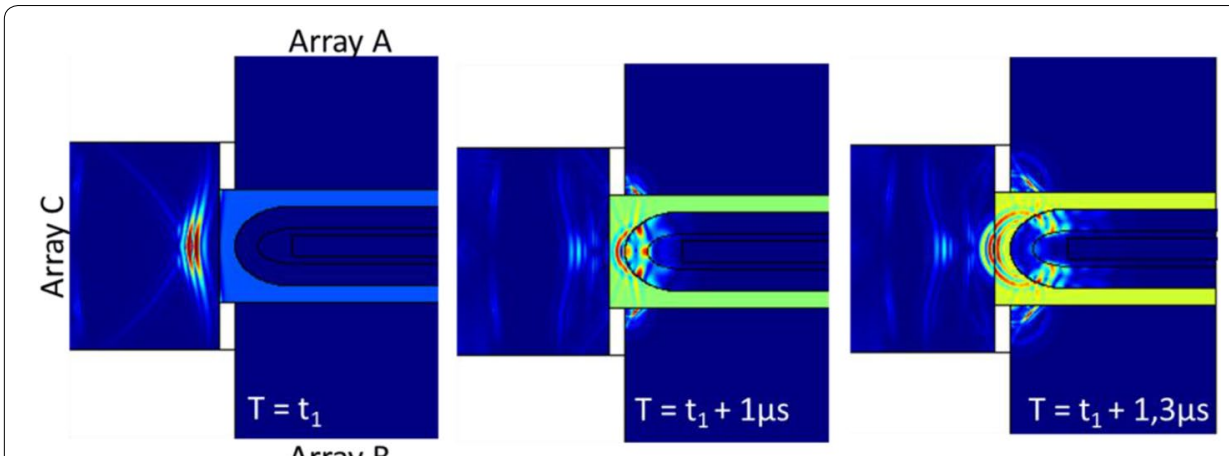

Fig. 5 Sound field simulation of the displacement value for three time points before and after the impact of the focused ultrasonic wave on seam loop

interact with any adhesive which may be present. There is no way to detect these wave packets through the array $C$. This effect increases with a seam loop that merges to a sharp corner.

A pulse-echo measurement using array $\mathrm{C}$ is therefore not useful according to the simulation and confirmed by test measurements. However, with a transmission measurement both the simulation and the real measurements reveal valuable differences in the amplitude images in defect and defect free areas. For this, the array A or B emits an ultrasound wave, which is tilted $26^{\circ}$ to the array $\mathrm{C}$ and which is subsequently detected by array $C$. The excitation is done by elements $1-6$ of array A or B to ensure that sufficient sound energy is introduced into the part. The tilt of the ultrasonic wave with an angle of $26^{\circ}$ results from the mathematical consideration, identical to [23], to excite a lamb wave in $\mathrm{S}$ mode in the outer sheet. The simulations confirm the stimulation of the lamb wave with that excitation setup (see Fig. 6). The detection is effected by averaging the signals form the six centre elements of array $\mathrm{C}$. The resulting amplitude images in case of a real measurement are shown in Fig. 7 for a completely filled with adhesive and air filled seam.

In Fig. 7, three characteristic echo sequences can be identified from the A scan of the real transmissions measurement. Through a parameter simulation study, the origins of the individual echoes can be determined. The echo sequence $S_{1}$ at approximately $12 \mu \mathrm{s}$ comes from multiple reflections of the excitation pulse on the sheet metal. The time displacement of the echo sequence $S_{2}$ for an air filled seam bond (at approx. $20 \mu \mathrm{s}$ ) and the echo from the sample with adhesive (at approx. $19 \mu \mathrm{s}$ ) can be explained by using simulations as displacement in the y-direction (see Fig. 1). The permitted displacements

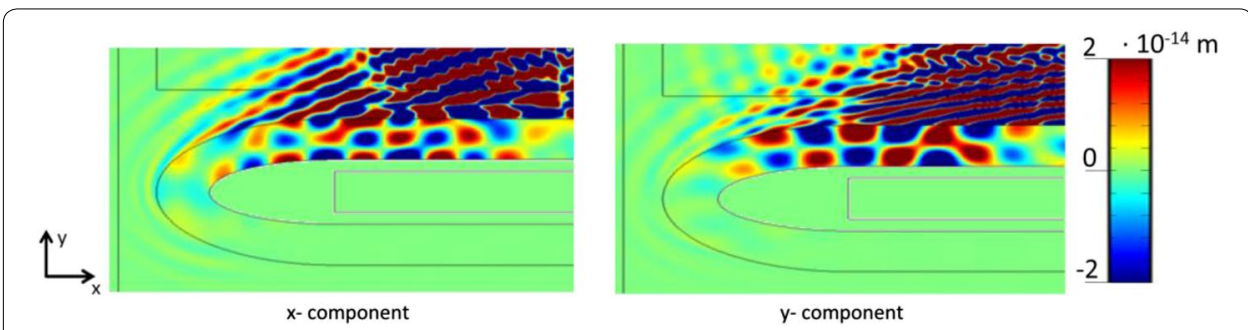

Fig. 6 Simulation of the displacement value in $x$, $y$ direction to provide the evidence of a propagation of the $S$ Mode lamb wave (seam bond completely filled with air) 


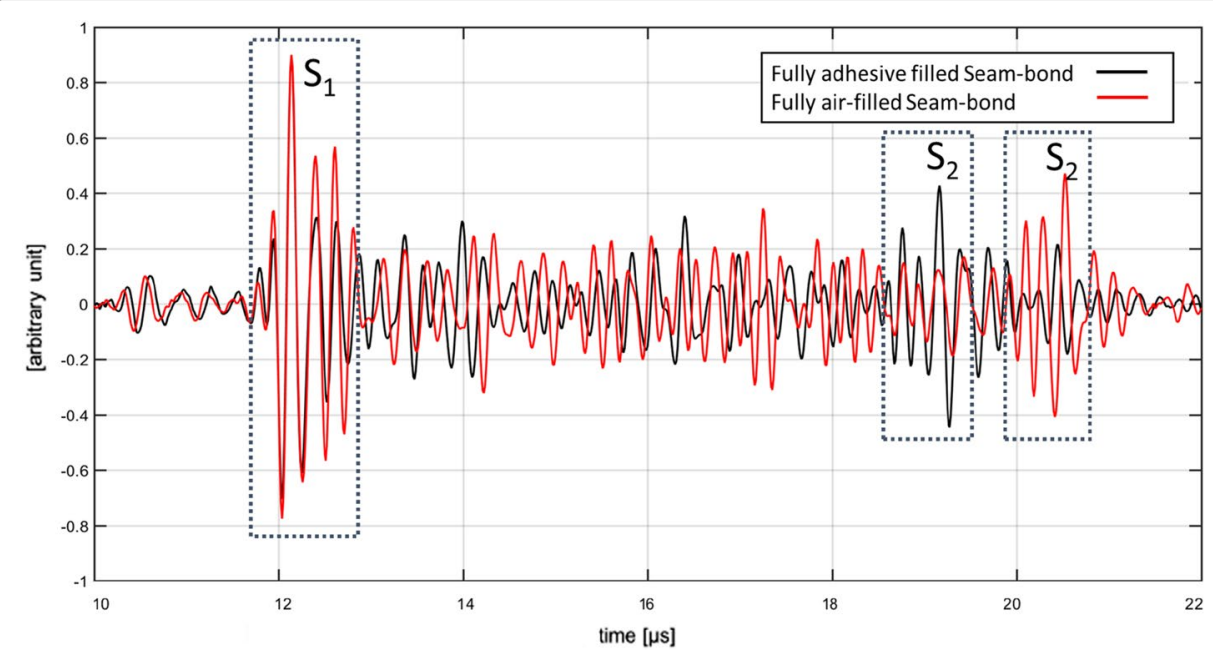

Fig. 7 Real Amplitude image of an unfocused wave (transmitter array A elements 4-12, receiver array C elements $7-10$ ) angled at $26^{\circ}$ to array $C$ on a seam bond completely filled with adhesive (black line) and completely filled with air (red line)

of s1 and s2, which are indirectly checked by the coupling conditions, must take into account for the evaluation range. The signal between the echoes at 12 and $19 \mu$ s can be explained by a formation of a lamb wave of S1 mode in the sheet metal (see Fig. 6). The lamb wave following the curvature of the seam radius whereby it is transferring energy into the wedge of the array $\mathrm{C}$.

\section{Influence of the seam tilting in the sensor}

In an inline inspection run with a robot guiding the device under test, a tilted seam in the sensor is not avoidable. The resulting effect is a decrease of the amplitude signal. For the results in Fig. 8, the simulated signal wave-form is evaluated in a gate G from 11 to $15 \mu \mathrm{s}$, whereby both possible evaluation methods for generating a $\mathrm{C}$ image are shown. For further considerations, the maximum amplitude or the integral energy of a simulation with a seam completely filled with adhesive and an angle of impact of the ultrasonic wave onto the sheet metal of 90 degrees is set to $100 \%$. The deviations caused by the defects and the tilted angle of the impact are given as a percentage.

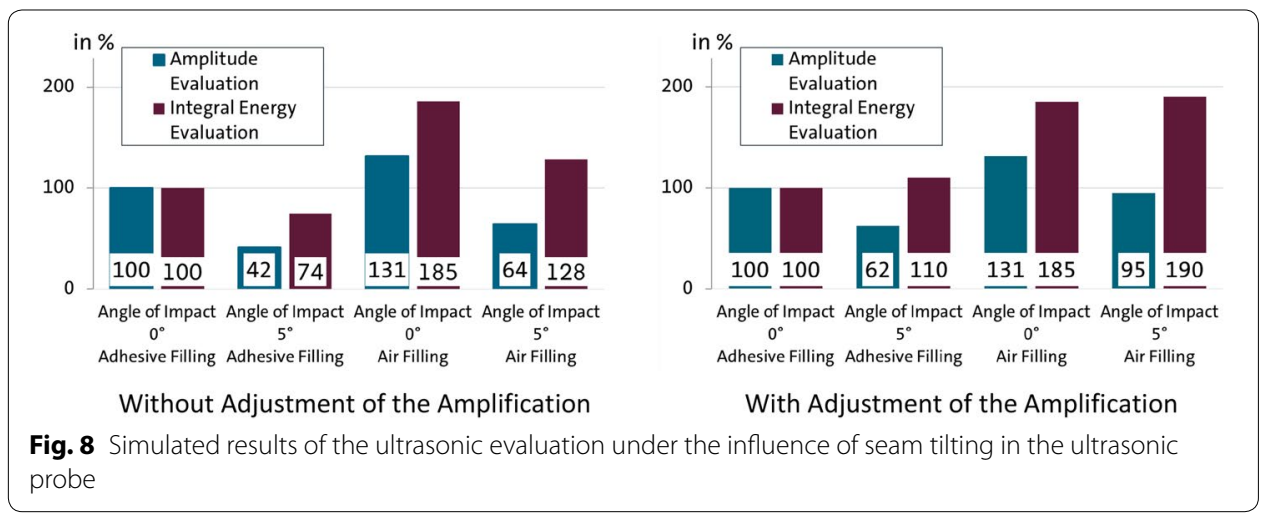


By means of these simulated A scans becomes clear, that a threshold value for the measurement regarding to the adhesive filling cannot be given under the influence of different angles of the impact. Through an adaption of the gain of the ultra sound signals, an evaluation with the integral energy method is possible. The total signal amplification is calculated from a fixed basic amplification and a variable gain component. This variable gain component is calculated from the amplification necessary to reach a maximum amplitude within a gate to a pre-set value. For the results in Fig. 8 right, the gate has been placed on the transition area from water to sheet metal. As the target value of the variable amplification the maximum amplitude is set to $90 \%$ of the total scale. The subsequent evaluation (see Fig. 8) of the new A Scan shows under identical evaluating-parameters that a threshold can be indicated better by the integral energy method. Therefore, the use of a variable gain is necessary to eliminate the tilting influence of the seam in the sensor.

\section{Experimental studies}

\section{Testing the seam bond areas $a$ and $b$}

To proof the new evaluation algorithm, samples with a Viton film replacing the adhesive are used to verify the smallest defect that can be detected. The samples are first examined using X-ray computer tomography to ensure that the film has not detached and lost contact with the outer sheet metal during the seam bonding process. In the sectional view of seam loop c in Fig. 9 a not binding to the outer sheet metal can be seen as a blue hole in the X-ray computer tomography. Consequently, for this sample the ultrasonic evaluation range is limited to seam areas $a$ and $b$.

In Fig. 10 an experiment with round artificial defects from 1 to $6 \mathrm{~mm}$ size is shown. Figure 10a shows the Viton film with laser cut round holes before integration into the seam bond. Figure 10b shows the result of the X-ray computed tomography after integration into the seam bond. The plane of the X-ray computer tomography examination is parallel to the sheet metal within the Viton film. The image plane is selected as close as possible to the outer sheet. Because of the blooming effects caused by the intensely differing absorption coefficients of the materials, the transition area from sheet metal to Viton foil cannot be represented by X-ray computed tomography. In Fig. 10c the coupling condition for array A is shown by evaluating the echo from the interface of water film and steel sheet. Figure 10d shows the ultrasound $\mathrm{C}$ images based on maximal

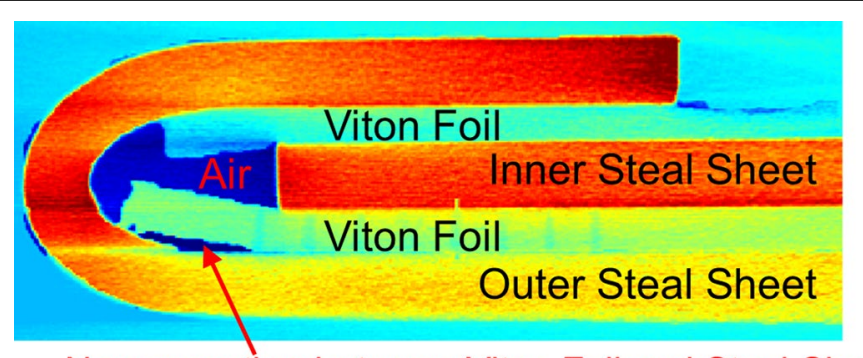

No connection between Viton Foil and Steal Sheet

Fig. 9 X-ray computer tomography in a false colour representation of a seam bonding with a Viton foil in the adhesive place 

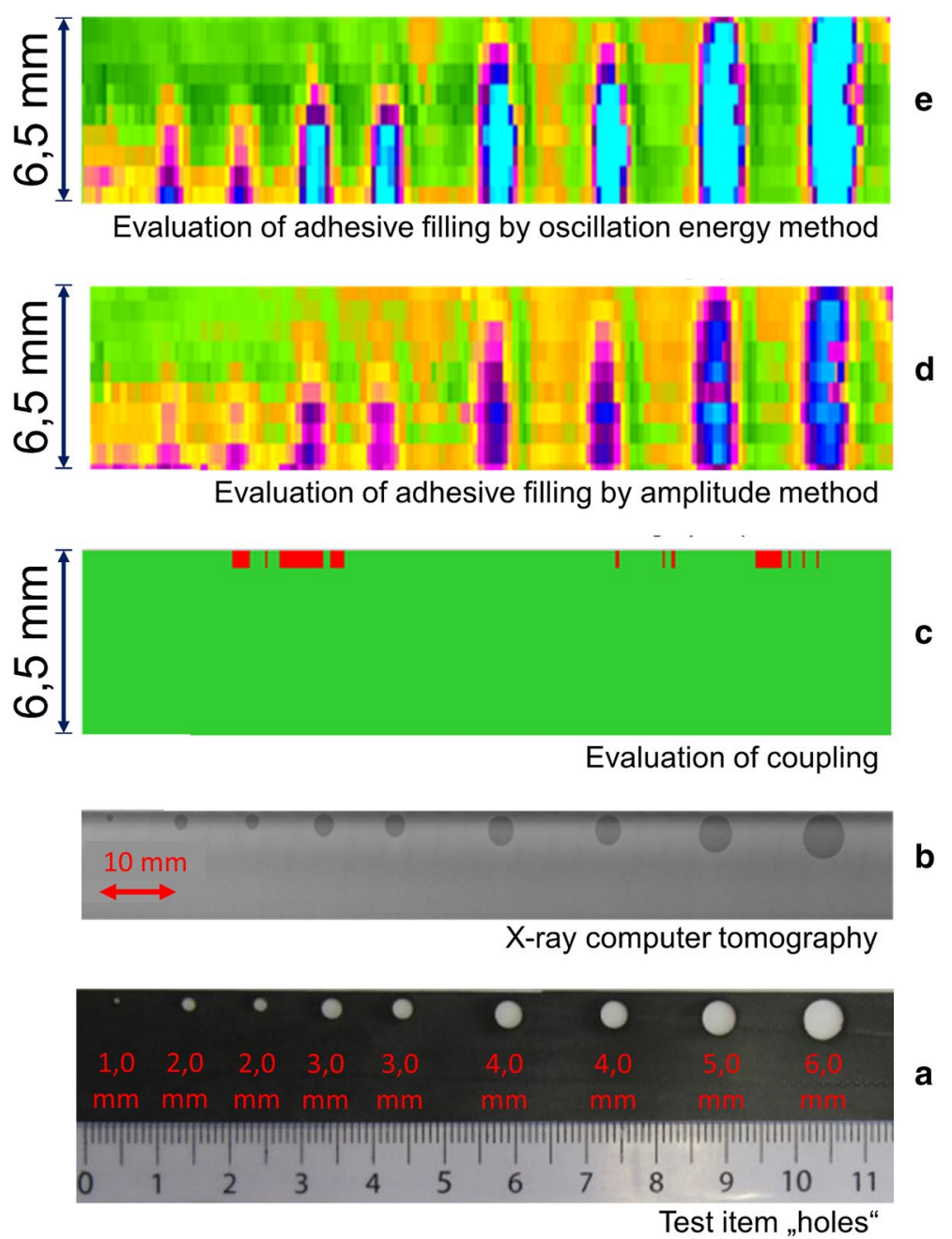

a

Fig. 10 Ultrasonic results of the sample with lased holes in a Viton foil in place of the adhesive to estimate the minimal error recognition. a Test item "holes", b X-ray computer tomography, c evaluation of coupling, $\mathbf{d}$ evaluation of adhesive filling by amplitude method, and e evaluation of adhesive filling by surface method

amplitude imaging. Finally Fig. 10e shows the result obtained by the integral energy evaluation. The time window for the evaluation range $\mathrm{G}$ for the amplitude and the integral energy evaluation is between 11 and $15 \mu$ s. The measurement is classified as valid if the coupling condition value exceeds a threshold of $65 \%$ at identical gain. If this is the case the coupling condition is marked as green. If the coupling amplitude is below $65 \%$ (red) the corresponding adhesive evaluation is not valid. If there is a strong curvature of the part or air bubbles are present in the water coupling conduction is typical not adequate and marked as red. All three ultrasonic evaluations are derived from the same raw measurement data from one inspection run. This single inspection run allows a direct comparison of the two data evaluation methods because external influence to the ultrasonic measurement (handling, temperature, etc.) can be excluded. 


\section{Testing the seam bond area c (seam loop)}

For testing the seam loop c a precisely teaching the robot path is necessary to have a constant position of the seam loop in the UT probe. However, the position of the seam bond in the inspection system can be checked using the ultrasonic signals. To determine the y-position of the metal sheet in the ultrasonic seam bond sensor (see Fig. 1), the elapsed time of the echoes from the water to steel transition of the two arrays A and B can be evaluated by using a defined time gate. This can also be done for the $\mathrm{x}$-position control by using a pulsed echo measurement of array $\mathrm{C}$. As both the robot path and the seam bond pack size are subject to deviations, a deviation of $0.4 \mathrm{~mm}$ in the $\mathrm{x}$ and $\mathrm{y}$ directions is considered tolerable. This tolerance window is set via the gate area. In the representation of the results in this document, this digital position evaluation is implemented using a red or green decision of the actual positional for validation of the measurement.

The Fig. 11 shows the ultrasonic results of a seam loop c combined with a destructive test of a laboratory sample with an adhesive filling. The ultrasonic excitation is done from the array A and array $\mathrm{B}$ respectively. The representation as a $\mathrm{C}$ image is performed by the integral energy evaluation within the gate $\mathrm{G}$ ranging from 14 to $18 \mu \mathrm{s}$ (see Fig. 7). The ultrasound results for the destructive test are shown for the seam areas a and b, depending on which array is providing the excitation. The coupling evaluation for the seam bond areas a, b and c is also shown. Thus, the test of the seam loop c consists of two ultrasonic measurements, excited by the array $\mathrm{A}$ and $\mathrm{B}$. Consequently, the $\mathrm{C}$ image of seam loop $\mathrm{c}$ has a resolution of two pixels for each probe position, whereby the base of the respective amplitude images is the transmission measurement by array $\mathrm{A}$ or array $\mathrm{B}$ onto array $\mathrm{C}$.

\section{Testing series production parts in the line}

Until this point, an idealised geometrical shape of the specimen was used for all samples examined. The samples exhibit constant radius of the seam loop and an unchanged adhesive layer thicknesses. This changes when series product parts are analysed. In this case, in addition to the previously mentions parameters, the orientation of the seam loop in the sensor also changes because the basis of the robot path are finite. Therefore, the tilting of the robot-guided part is unavoidable. This influence can be controlled through the new automated adjustment of the gain. The aim, as shown in the simulations, is to compensate the effect of the tilting angle of the part within the sensor. In the present

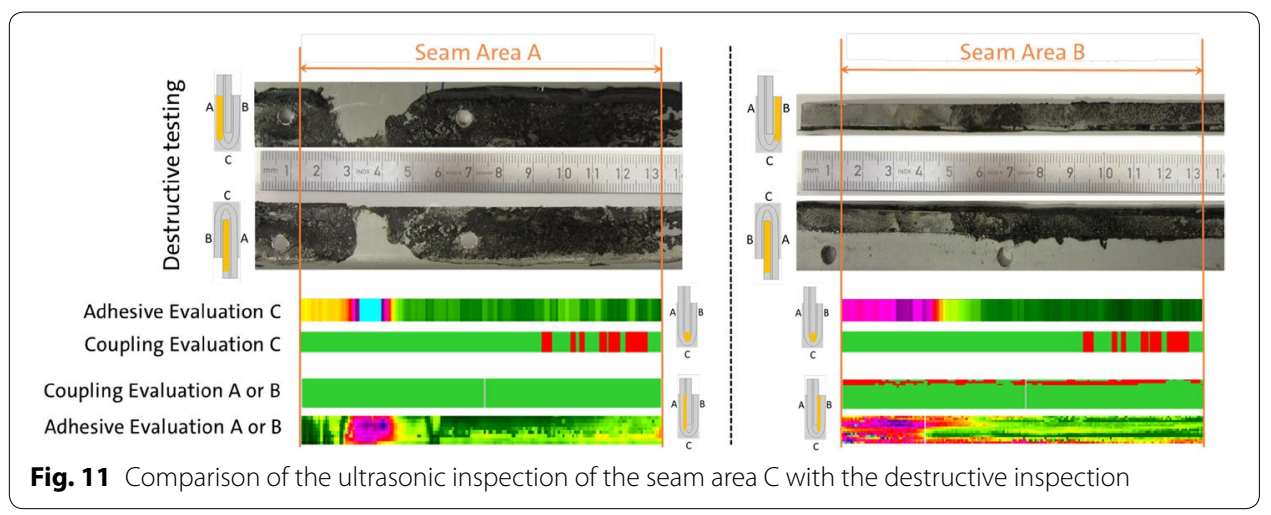


study the gate has been placed on the transition area from water to sheet metal. As the target value of the variable amplification the maximum amplitude is set to $90 \%$ of the total scale. This additional gain is applied to the complete amplitude signal of every aperture.

The seam bond inspection system has been integrated as the last station in the luggage compartment door production system. The inspection speed, means the speed the robots moves the door thru the seam bond sensor is actual set to $30 \mathrm{~mm} / \mathrm{s}$. Due to time restriction by the cycle time and time needs for handling the part the achievable inspection length is approximately $1 \mathrm{~m}$ in one cycle step of $90 \mathrm{~s}$. Consequently, a complete inline check is not possible in the actual state. Instead, different over-lapping sections of seam bond areas are tested in successive luggage compartment doors so that the complete seam has been tested after four successive luggage compartment doors.

Figure 12 shows results for validating the inspection method in serial part production. The results show a seam bond area a with two test areas. Area 1 of the sample that underwent destructive testing exhibits predominantly defect-free adhesion. Only two meander structures, recognisable by the darker blue of the adhesive, are located in the right-hand section of test area 1 . In the area 2 a number of meander structures occur.

The non-destructive test systems returns a similar result. But a large defect can be seen in the left-hand section of the result image for area 1. However, the coupling evaluation shows a red coupling exactly for this area so that no adhesive evaluation can be given at this point. Reason for the red coupling could be air bubbles in the water layer or an overly strong tilting of the seam in the sensor. In area 2 of the Fig. 12 the destructive test reveals several meander structures. This is indicated by the yellow or red pixels in the ultrasound C Scan. However, the spatial resolution of the ultrasound system is less than the optical inspection of the opened seam bond. This is the reason why defects in the $\mathrm{C}$ Scan of the ultrasonic system look different and are more clustered.

\section{Discussion of the results}

\section{Seam areas $\mathbf{a}$ and $\mathbf{b}$}

From the ultrasound results of the sample with a Viton foil replacing the adhesive it can be seen that defects with a diameter of $2 \mathrm{~mm}$ can no longer be identified with the commonly used amplitude evaluation. In contrast, $2 \mathrm{~mm}$ defects are reliably identified when
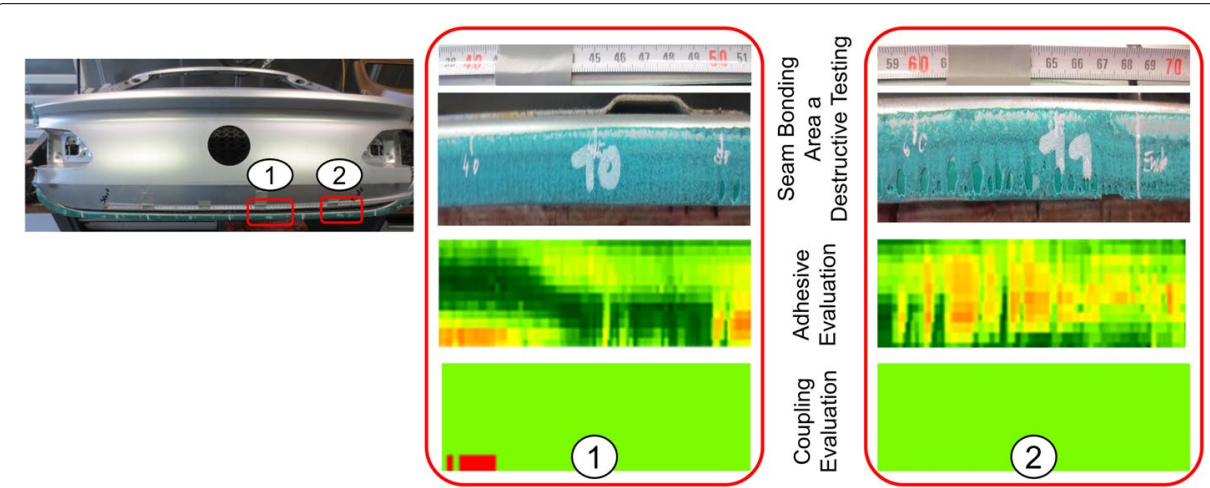

Fig. 12 Result of an inline ultrasound testing of real production parts combined with destructive inspection for validation 
evaluating the amplitude signals using integral energy evaluation. However, a hole with a diameter of $1 \mathrm{~mm}$ can not identified as a defect even when using integral energy evaluation. In contrast to the amplitude method, the integral energy method shows a better contrast between imperfect areas and those with Viton foil. Since the probe geometry and the defects are idealised, real defects with a diameter of $1 \mathrm{~mm}$ cannot be recognized by the ultrasonic system. Nevertheless it can be shown (see Fig. 12), that meander structures in serial production parts can be identified because of the cumulative occurrence. As a consequence the integrated failure rate increases.

The identification of small defects and the greater contrast when using integral energy evaluation is due to the higher information content of the evaluated data and the better signal-to-noise ratio. An amplitude outlier therefore does not necessarily lead to an erroneous evaluation as this has less of an effect on the overall measurement result. The higher information content of the evaluated data is due to the volume of data that is evaluated. For the selected gate $\mathrm{G}$ of $4 \mu \mathrm{s}(11-15 \mu \mathrm{s})$ and the sampling rate used of 80 $\mathrm{MS} / \mathrm{s}$, the values of 320 individual amplitudes are combined into one evaluation. With the amplitude evaluation, in contrast, only the highest sampled value within a gate is evaluated.

The benefit in information is illustrated with the already known results of the sound field simulations and with one example using two measuring points in Fig. 4. The real measurements are an average value with an associated confidence interval of ten repeated measurements. The two measuring points are located in a laboratory sample. A Universal robot was used to manipulate the sample to ensure that the identical points are examined by the ultrasound testing system. The specification states that this type of robot has a repeatability of $\pm 0.1 \mathrm{~mm}$ [24]. The resulting amplitude images were then evaluated and averaged according to the amplitude or integral energy evaluation. The deviations between a seam completely filled with adhesive and air in the simulation were not achieved in the real measurements, but the difference in the integral energy evaluation is greater. This justifies a higher probability of finding even small defects and a better contrast when using the integral energy evaluation. The smaller confidence intervals in the integral energy method demonstrate the greater robustness of this evaluation method.

\section{Seam area c}

Figure 11 shows the result of the ultrasonic examination for determining the degree of filling of seam loop C. Influences due to a deviation of the seam loop position in the sensor can be excluded because of the good coupling. The red marked areas in coupling evaluation in the upper seam bond area $\mathrm{b}$ arises due to the larger active sensor area $(6.5 \mathrm{~mm})$ in contrast to the flange length of seam area $\mathrm{b}(6 \mathrm{~mm})$. Consequently, the inner sheet is sounded in this area, whereby the echo resulting from the material transition is generated outside the gate for an OK coupling. A change in the orientation of the seam bond loop of the sample within the sensor can be excluded as a further factor influencing the result. For this purpose, the straight edge of the laboratory sample has been investigated in this series of tests. Consequently, it can be ensured that the ultrasound results are solely related to the adhesive distribution and not to the geometrical shape of the seam bond or the orientation of the seam loop. Further investigations must show the extent to which this can be ensured later in the series process. 
It can be seen from Fig. 11 that the ultrasound results for the evaluation of seam loop c and the areas $\mathrm{a}$ and $\mathrm{b}$ correlate. Consequently, the transmission measurement from array A to array $\mathrm{C}$ shows a defect at a bond position between 3 and $4 \mathrm{~cm}$. The destructive test shows a large defect in area $a$ in this seam section. In the area $b$, the transmission measurement from the array $B$ to the array $C$ shows a defect at the position between 1.5 and $5 \mathrm{~cm}$. Here too, the destructive test shows a significantly reduced amount of adhesive than in the following areas. However, the destructive test shows that test area $\mathrm{c}$ is filled with adhesive over the entire sample length.

The cause of this false evaluation of the adhesive by the ultrasound system can be explained by the path of the ultrasonic wave along the outer sheet. To achieve sufficient sound energy and to compensate for possible losses due to air bubbles in the water coupling, the first six elements of arrays A or B sound towards the adjacent array C. Disregarding the opening angle of the transmitted ultrasonic wave and any present side lobes, an area of $2.3 \mathrm{~mm}$ is excited in the front part of the seam bond, whereas micro sections of the laboratory samples show the length of the seam channel to be approximately $0.8 \mathrm{~mm}$. As a result, the predominant part of the sound path lies in the seam areas a and b, where the ultrasonic wave interacts with any adhesive which may be present. A shortening of the propagation path within seam areas $a$ and $b$ can be achieved by reducing the elements emitting the sound. However, this is associated with a significant decrease in signal stability and an accompanying reduction in the repeatability of the measurement.

The sound field simulations can also demonstrate the relationship between the transmission measurement for the evaluation of the seam loop $\mathrm{c}$ and the degree of adhesive filling in seam areas a or b. The simulation results show that under identical excitation, detection and evaluation range the integral energy method within a gate $G$ of a seam completely filled with air is 2.1 times greater than in the case of a seam bond completely filled with adhesive. This deviation between OK and not okay bonding is already reduced to 1.4 times if the defect area only extends to seam loop C. Subsequently, the selective evaluation of seam loop $\mathrm{C}$ by transmission measurement is only possible if this result is used in connection with the results of the pulse echo measurement of seam areas a or b. Consequently there are two conditions for the test of the adhesive filling of the seam loop C. First the position in $\mathrm{x}$ and $\mathrm{y}$ direction of the seam in the sensor must be within a certain range $( \pm 1 \mathrm{~mm})$. This is ensured by three impulse echo measurements of the arrays A, B and C. The second condition assumes a complete filling of the seam areas $\mathrm{a}$ and $\mathrm{b}$. This is checked by the ultrasonic system. Only if both conditions for a test position are fulfilled, the validation of the seam area $\mathrm{c}$ is possible. In the present case, a negative adhesive evaluation for areas a and $b$ has been established by the pulse echo measurement of arrays A and B. Subsequently, because of the relationship between the transmission measurement and the degree of adhesive filling of areas $a$ and $b$, no result of the transmission measurement for the evaluation of seam loop c can be indicated for this test position.

\section{Series production parts}

The detection of defects within the adhesive can be proofed by the testing of series production parts in the line. These are the most difficult defects for the testing method to detect as the ultrasonic wave penetrates the adhesive depending on the ratio of the 
sound resistance and is also attenuated. The reason is that, in contrast to the destructive test, the position of the flaws in area 2 of Fig. 12 in the $\mathrm{C}$ image migrates in its height (y-direction), can be attributed to the position of the seam loop in the UT probe. This can be presupposed as non-uniform due to the robot's path. The reason that the defects are not separately represented as such is due to the spatial resolution of the UT probe. The transducer length of $8 \mathrm{~mm}$ and an aperture consisting of 3 adjacent elements excites an area of $8 \mathrm{~mm} \times 1.15 \mathrm{~mm}$, neglecting the divergence angle. The following evaluation is integrated over this area, resulting in a superimposition of adjacent meander structures. The consequence is that due to the $8 \mathrm{~mm}$ long extension of the transducers in the z-direction (see Fig. 1 on the right) several defects are ultrasonically superimposed. Therefore, these flaws cannot be displayed separately in the $\mathrm{C}$ image.

The use of a coupling condition is shown to be particularly useful where the geometrical shape of the component is not level. Without simultaneous evaluation of the coupling condition, a defect in the left test area 1 would be detected in the ultrasound result (see Fig. 12). However, in this area the coupling shows a red evaluation so that the evaluation of the adhesive assessment is not possible due to the position or orientation of the seam bond in the sensor. In a further software development step, the pixels in the adhesive evaluation are greyed out where there is a negative coupling condition.

In a pilot run based on an 5 month measurement campaign 78\% (79\% seam bond area b) of the errors detected by a weekly conducted destructive test were identified by the phased array system in the seam bond area a. In 1\% (4\% seam bond area b) of the measurements, the coupling was insufficient, so that for this part of the seam no adhesive evaluation can be given. The deviation from $21 \%$ (17\% seam bond area $\mathrm{b}$ ) is partially be explained by the fact, that the two testing methods do not show the same results. For the detection of errors with the destructive testing, these errors must be located in the abort edge. That this is not the case for every error can be shown by micro sections after the destructive test. Thus, only the error positions 3 and 4 can be detected by the destructive test (see Fig. 13). Conversely, the ultrasonic system detects the error positions 1 and 3 and the error positions 2, 4 and 5, depending on the size of the defect. Therefore, discrepancies between these two methods are inevitable. Furthermore, the identification of fewer errors with the destructive test is querying the actual testing procedure of seam bonds.

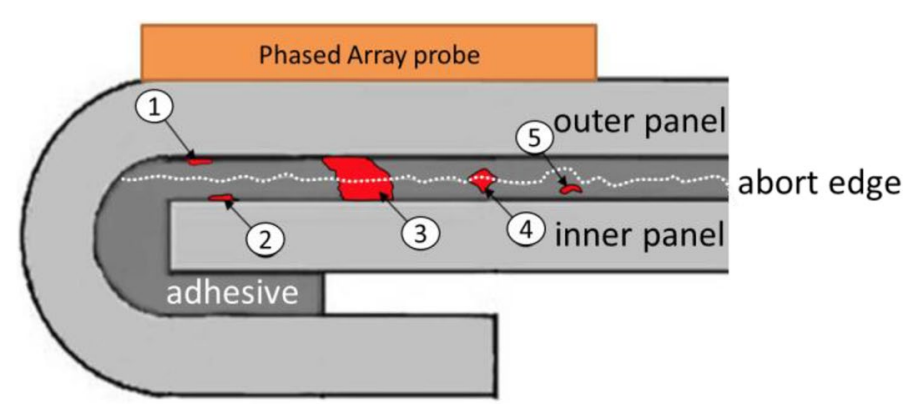

Fig. 13 Comparison the results of destructive and nondestructive testing of seam bonds 


\section{Conclusion and further steps}

For the idealised samples with laser-cut holes, the new integral energy evaluation shows an improvement in defect detectability. Furthermore, the contrast could be enhanced unlike in the conventional amplitude evaluation. The reasons for this lie in the higher information content and averaging of the underlying number of data, which increases with a larger gate width. However, if the gate area is too large, smaller defects are no longer recognised. The temporally rear oscillations amplitudes are quite as high as the background noise. Therefore, no more value is added. A large number of measurements and simulations have revealed that an evaluation of 18 oscillations, which corresponds to approx. $4 \mu \mathrm{s}$, has been established as a good compromise.

The approach has also been discussed for inspection of all three seam areas using a multi-phased array ultrasound system. The examination of the seam area a and $b$ is possible using the pulse echo method and evaluation by using the integral energy method. A transmission measurement has been discussed as an evaluation method for seam area $\mathrm{C}$. In this method array A or B emits a tilted ultrasonic wave, which is detected by array $\mathrm{C}$. The resulting difference in the amplitude images can be better resolved by using the new integral energy evaluation than by the conventional amplitude evaluation. In addition to the defined position and orientation range of the test specimen in the sensor shaft, an influence of the adhesive distribution in seam area a or b has also been observed on the transmission measurement result. A more selective evaluation of seam area c can be achieved by reducing the number of exciting elements, which in turn has a negative effect on the signal stability. The discussed combination of the adhesive evaluation for area $\mathrm{a}$ and $\mathrm{b}$ with the transmission measurement is promising. The implementation of this functionality in the evaluation software is currently being sought.

The inline measurement of the seam areas $a$ and $b$ in prototypical series operation is currently being performed. For this application, it has been shown that defects within the adhesive bond are identified. In area $b$ the limit of the adhesive trace can also be recognised. This is useful as an input parameter for optimisation purposes, for example, for adapting the adhesion application. This eliminates the need for destructive tests. The automated evaluation has already been implemented within the software for determining the degree of filling of the seam bond. This is evaluated by means of a pixel evaluation. Therefore, a statistic can be provided to the productions sites concerned, to identify trends and, in particular, permanently faulty areas. Efforts must still be made in the consideration of the defect position. An open channel detected within the seam areas a or b, which enables the penetration of moisture into the joining plane, must necessarily trigger an not in order evaluation. This functionality has been implemented in the software in a further development step. Furthermore, the array A has to be enlarged to cover the entire seam bond area a.

Authors' contributions

MK performed the simulations and the experiments. MK wrote the manuscript. HH helped to write the manuscript. Both authors read and approved the final manuscript.

Author details

1 Division Technology Management, Volkswagen AG, 38440 Wolfsburg, Germany. ${ }^{2}$ Division Director Testing Systems, Fraunhofer Institute for Ceramic Technologies and Systems, 01109 Dresden, Germany. 


\section{Competing interests}

The authors declare that they have no competing interests.

Availability of data and materials

All relevant data of the article are presented in the manuscript.

\section{Consent for publication}

The authors consent to the publication process.

Ethics approval and consent to participate

The authors consent to the ethics.

Funding

Not applicable.

\section{Publisher's Note}

Springer Nature remains neutral with regard to jurisdictional claims in published maps and institutional affiliations.

Received: 15 October 2017 Accepted: 9 December 2017

Published online: 21 December 2017

\section{References}

1. Yoon Y, Shin D, Kim D, Kwak H, Ryu Y, Han B. The study of roller hemming process for aluminum alloy via finite element analysis and experimental investigations. SAE technical paper series: SAE International 400 Commonwealth Drive. Warrendale; 2007.

2. Habenicht Gerd. Applied adhesive bonding: a practical guide for flawless results. Weinheim: Wiley-VCH Verlag $\mathrm{GmbH} \&$ Co. KGaA; 2008.

3. Neugebauer R, Jesche F, Menzel S, Richter K, Mayer B, Fricke H, Neumann I. Falzkleben im automobilen Rohbau. Hochfeste und hybride Materialien. 2011;32:425-41.

4. Wanintradul C, Golovashchenko SF, Gillard AJ, Smith LM. Hemming process with counteraction force to prevent creepage. J Manuf Process. 2014;16(3):379-90. https://doi.org/10.1016/j.jmapro.2014.04.003.

5. Brockmann Walter, Gei Paul L, Klingen Jrgen, Schrder Bernhard. Adhesive bonding: materials, applications and technology. Weinheim: Wiley-VCH Verlag GmbH \& Co. KGaA; 2008.

6. Bahloul R, Ben-Elechi S, Potiron A. Optimisation of springback predicted by experimental and numerical approach by using response surface methodology. J Mater Process Technol. 2006;173(1):101-10. https://doi.org/10.1016/j. jmatprotec.2005.11.009.

7. Woyke W, Jesche F, Menzel S, Landgrebe D. Das Falzkleben fehlerfrei gestalten. adhäsion KLEBEN \& DICHTEN. 2017:61(12):16-9. https://doi.org/10.1007/s35145-017-0002-1.

8. Livatyali H, Larris SJ. Experimental investigation on forming defects in flat surface-convex edge hemming: roll, recoil and warp. J Mater Process Technol. 2004;153-154:913-9.

9. German Institut for Standardisation. Adhesice bonding technology — quality requirements for adhesive bonding processes_-Part 1: adhesive bonding process chain, no. DIN EN 2304-1: Beuth Verlag; 2016. Accessed September 01 2017.

10. Wiese E. Kleb- und prozesstechnische Bewertung des Falzklebens. 1. Forschungsberichte des Instituts für Füge-und Schweißtechnik 37. Aachen: Shaker; 2015.

11. Maev RG, Titov S, Bogachenkov A, Ghaffari B, Lazarz K. Evaluation of adhesively bonded joints in automotive industry with ultrasonic matrix array transducer. Cancun Mexico, October 2; 2011.

12. Korzeniowski M, Piwowarczyk T, Maev RG. Application of ultrasonic method for quality evaluation of adhesive layers. Arch Civil Mech Eng. 2014;14(4):661-70.

13. Shui G, Wang YS, Huang P, Qu J. Nonlinear ultrasonic evaluation of the fatigue damage of adhesive joints. NDT E Int. 2015;70:9-15.

14. Wiese E, Eichleiter F. Study of production influence on the property profile on fold bondings. Bad Nauheim: Joining in Car Body Engineering; 2012. p. 2012.

15. Titov Sergey A, Maev Roman G, Bogachenkov Alexey N. Pulse-echo NDT of adhesively bonded joints in automotive assemblies. Ultrasonics. 2008;48(6-7):537-46. https://doi.org/10.1016/j.ultras.2008.07.001.

16. Maeva EY, Severina IA, Bugaev AS, Severin M, Maev RG. Acoustical imaging evaluation of thin metal-metal adhesive bonds. In: IEEE symposium on ultrasonics; 2003. p 2037-40

17. Adams RD, Drinkwater BW. Non-destructive testing of adhesively-bonded joints. IJMPT. 1999;14(5/6):385. https://doi. org/10.1504/IJMPT.1999.036279.

18. Multiphysics COMSOL. Comsol multiphysics user guide (version 5.2). COMSOL, AB. 2015. p 5-437.

19. Kato D, Han D. Volumen viscosity and it-induced bulk modules of heavy oil. In: 71. EAGE conference and exhibition incorporating SPE EUROPEC; 2009

20. Thuillier S. Numerical simulation of the hemming process in the case of Al alloys. In: AIP conference proceedings. AIP; 2005. p 691-98.

21. Sika Automotive GmbH. SikaPower 492G: Semi crash resistant hem flange adhesive. Product data sheet. 2009. http://zaf.sika.com/dms/getdocument.get/aee2b39e-02c6-3781-9611-5cee923a9002/PDS\%20SikaPower-492G\%20 english\%2002-2009.pdf. Accessed 2 Sept 2017. 
22. Rice SO. Mathematical analysis of random noise. Bell Syst Tech J. 1944;23(3):282-332. https://doi. org/10.1002/j.1538-7305.1944.tb00874.x.

23. Hoever C, Prager J, Brekow G, Kreutzbruck M. Fehlernachweis mit geführten Wellen unter Verwendung der Gruppenstrahlertechnik: Münster, 18-20. Mai 2009; DGZfP-Jahrestagung 2009 Zerstörungsfreie Materialprüfung. In: DGZfP-Jahrestagung 2009. 115-CD. 2009.

24. Universal Robots. Technical Specifications UR 10. 2015. https://www.universal-robots.com/products/ur10-robot/. Accessed 2 Sept 2017

Submit your manuscript to a SpringerOpen journal and benefit from:

- Convenient online submission

Rigorous peer review

Open access: articles freely available online

- High visibility within the field

- Retaining the copyright to your article

Submit your next manuscript at $\boldsymbol{\Delta}$ springeropen.com 